\title{
On invariant projectively flat affine connections
}

(Dedicated to Professor Fuichi Uchida on the occasion of his sixtieth birthday)

\author{
Hajime URAKAWA
}

(Received August 29, 1997; Revised June 1, 1998)

\begin{abstract}
We give a simple characterization of invariant projectively flat affine connections, and classify all irreducible Riemannian symmetric spaces with invariant projectively flat affine connections, and classify all simple Lie groups with left invariant projectively flat affine connections with some exceptions. These works accomplish an early Agaoka's work (cf. [1]) which gave many examples of the classical cases.
\end{abstract}

Key words: projectively flat, affine connection, invariant connection, symmetric space, real representation.

\section{Introduction}

Recently, Shima (cf. [13]) characterized invariant projectively flat affine connections in terms of affine representations of Lie algebras, and showed that a homogeneous space admits an invariant projectively flat affine connection if and only if it has an equivariant centro-affine hypersurface immersion.

Let $M=G / K$ be a homogeneous space with a connected Lie group $G$ and a closed subgroup $K$, and let $\mathfrak{g}, \mathfrak{k}$ the corresponding Lie algebras. A finite dimensional real representation $(f, \widetilde{V})$ of the Lie algebra $\mathfrak{g}$ is called to be spherical relative to a pair $(\mathfrak{g}, \mathfrak{k})$ if there exists a non-zero vector $v_{0} \in \widetilde{V}$ satisfying

$$
f(X) v_{0}=0, \quad \forall X \in \mathfrak{k} .
$$

Then our first main theorem is

Theorem A (cf. Theorem 1.3) A homogeneous space $M=G / K$ admits a $G$-invariant projectively flat affine connection if and only if there exists a real representation $(f, \widetilde{V})$ of $\mathfrak{g}$ of dimension $\operatorname{dim} M+1$, which is spherical relative to $(\mathfrak{g}, \mathfrak{k})$ and satisfies

1991 Mathematics Subject Classification : Primary, 53A15; Secondary, 53C35, 53C42, $22 \mathrm{E} 45$. 


$$
\widetilde{V}=f(\mathfrak{g}) v_{0} \oplus \mathbf{R} v_{0}
$$

As its application, we obtain the following classification theorems:

Theorem B (cf. Theorem 2.2) Let $M=G / K$ be an irreducible simply connected Riemannian symmetric space. Then $M=G / K$ admits a $G$ invariant projectively flat affine connection if and only if $M=G / K$ is one of the following:

(1) $S O(n+1) / S O(n), n \geq 2$,

(2) $S L(n+1, \mathbf{R}) / S O(n+1), n \geq 2$,

(3) $S U^{*}(2 n) / S p(n), n \geq 3$

(4) $S O_{o}(n, 1) / S O(n), n \geq 2$,

(5) $S L(n+1, \mathbf{C}) / S U(n+1), n \geq 1$,

(6) $E_{6} / F_{4}$ (non-compact type of EIV).

Moreover, we obtain:

Theorem C (cf. Theorem 3.1) Let $G$ be a real simple Lie group. If $G$ admits a left invariant projectively flat affine connection, then $\mathfrak{g}$ is one of the following:

(a) $\mathfrak{o}(3) \cong \mathfrak{s u}(2)$

(b) $\mathfrak{s l}(n+1, \mathbf{R}), n \geq 1$,

(c) $\mathfrak{s u}^{*}(2 n), n \geq 2$,

(d) $\mathfrak{s u}(r, s)(r+s=$ even, $r+s \geq 4) ; \mathfrak{o}(3,4) ; \mathfrak{o}(1,9), \mathfrak{o}(5,5) ; \mathfrak{o}(3,11)$, $\mathfrak{o}(7,7)$.

We remark in Theorem $\mathrm{C}$ that in the cases $(\mathrm{a}) \sim(\mathrm{c}), G$ admits a left invariant projectively flat affine connection, but for the case (d), we do not know whether or not $G$ admits such a connection.

\section{Invariant projectively flat affine connections}

We first describe Shima's theorem characterizing projectively flat affine connections following [13], [11] and [12].

Let $D$ be an affine connection on an $n$-dimensional $C^{\infty}$ manifold $M$. We always assume that $D$ is $C^{\infty}$, torsion-free and Ricci-symmetric (cf. [11]).

The connection $D$ is called to be projectively flat if in a neighborhood of each point in $M, D$ is projectively equivalent to an affine connection $\bar{D}$ which is flat, that is, the curvature tensor $\bar{R}$ is identically zero. It is well-known that $D$ is projectively flat if and only if 
(i) the curvature tensor $R$ of $D$ satisfies

$$
R(X, Y) Z=\frac{1}{n-1}\{\operatorname{Ric}(Y, Z) X-\operatorname{Ric}(X, Z) Y\},
$$

where Ric is the Ricci tensor, and

(ii) Ric satisfies the Codazzi equation, that is,

$$
\left(D_{X} \operatorname{Ric}\right)(Y, Z)=\left(D_{Y} \operatorname{Ric}\right)(X, Z),
$$

for all tangent vectors $X, Y, Z$ (see [11] or [12]).

Let $M=G / K$ be a homogeneous space of a connected Lie group $G$ and a connected closed subgroup $K$ of $G$. We assume $G$ acts effectively on $G / K$ and $M=G / K$ is simply connected. We denote by $\mathfrak{g}$ and $\mathfrak{k}$, the Lie algebras of $G$ and $K$, respectively. We enlarge $\mathfrak{g}$ to $\widetilde{\mathfrak{g}}$ as follows:

$$
\left\{\begin{array}{l}
\widetilde{\mathfrak{g}}=\mathfrak{g} \oplus \mathbf{R} E, \\
{[\widetilde{\mathfrak{g}}, E]=\{0\} .}
\end{array}\right.
$$

Then Shima (cf. [13]) obtained the following theorem:

Theorem 1.1 A homogeneous space $M=G / K$ admits a $G$-invariant projectively flat affine connection if and only if $\tilde{\mathfrak{g}}$ has an affine representation $(\tilde{f}, \widetilde{q})$ on a real affine space $\tilde{V}$, that is,

(i) $(\tilde{f}, \widetilde{V})$ is a real representation,

(ii) $\widetilde{q}$ is an $\mathbf{R}$-linear mapping from $\widetilde{\mathfrak{g}}$ into $\tilde{V}$ such that

$$
\widetilde{q}([\tilde{X}, \tilde{Y}])=\tilde{f}(\tilde{X}) \widetilde{q}(\tilde{Y})-\tilde{f}(\tilde{Y}) \widetilde{q}(\tilde{X}), \quad \text { for } \tilde{X}, \tilde{Y} \in \tilde{\mathfrak{g}},
$$

with the following properties:

(iii) $\operatorname{dim} \tilde{V}=\operatorname{dim} M+1$,

(iv) $\widetilde{q}$ is surjective and the kernel is $\mathfrak{k}$,

(v) $\widetilde{f}(E)$ is the identity mapping of $\widetilde{V}$ and $\widetilde{q}(E) \neq 0$.

Remark 1.2 In Theorem 1.1, we need not the assumption of simply connectedness of $G$ itself. Indeed, we take $\widetilde{G}$ the universal covering Lie group of $G$ with the projection $\pi: \widetilde{G} \longrightarrow G$, and $\widetilde{K}$ be the identity component of $\pi^{-1}(K)$. Then we have $M=G / K=\widetilde{G} / \widetilde{K}$ because of simply connectedness of $\widetilde{G} / \widetilde{K}$ and $M$. Moreover, since a translation by each element in $G$ coincides with some translation by an element in $\widetilde{G}$, a $\widetilde{G}$-invariant projectively flat affine connection on $M$ guaranteed by the original Shima's theorem is also $G$-invariant. 
Now Theorem 1.1 can be restated as follows:

Theorem 1.3 A homogeneous space $M=G / K$ admits a $G$-invariant projectively flat affine connection if and only if there exists a real representation $(f, \widetilde{V})$ of $\mathfrak{g}$ of dimension $\operatorname{dim} M+1$, which is spherical relative to $(\mathfrak{g}, \mathfrak{k})$ and satisfies

$$
\widetilde{V}=f(\mathfrak{g}) v_{0} \oplus \mathbf{R} v_{0}
$$

Proof. Theorem 1.3 can be obtained from Theorem 1.1. Substituting $\widetilde{X}=E$ in the equation in (ii) of Theorem 1.1, we have

$$
\widetilde{q}(\tilde{Y})=\tilde{f}(\tilde{Y}) \widetilde{q}(E), \quad \tilde{Y} \in \widetilde{\mathfrak{g}},
$$

since $[E, \tilde{Y}]=0$ and $\tilde{f}(E)$ is the identity mapping of $\widetilde{V}$. Let $v_{0}=\widetilde{q}(E)$ be a non-zero element in $\widetilde{V}$ by (v). By (iv) and (1.4), for $\tilde{Y} \in \mathfrak{k}$,

$$
\tilde{f}(\tilde{Y}) v_{0}=0
$$

We denote by $f$ the restricted representation to $\mathfrak{g}$ of $\tilde{f}$. Then (1.5) implies that $(f, \tilde{V})$ is a spherical representation of $\mathfrak{g}$ relative to $(\mathfrak{g}, \mathfrak{k})$. By (iii) and (iv), we have

$$
\widetilde{V}=f(\mathfrak{g}) v_{0} \oplus \mathbf{R} v_{0}
$$

thus we obtain the desired spherical representation $(f, \widetilde{V})$.

Conversely, assume that $(\mathfrak{g}, \mathfrak{k})$ admits a real representation $(f, \widetilde{V})$ satisfying the conditions of Theorem 1.3. We first enlarge $\mathfrak{g}$ to $\widetilde{\mathfrak{g}}=\mathfrak{g} \oplus \mathbf{R} E$ with $[E, \mathfrak{g}]=\{0\}$ and enlarge $f$ to a representation of $\widetilde{\mathfrak{g}}$ by

$$
\left\{\begin{array}{l}
\tilde{f}(X)=f(X), \quad X \in \mathfrak{g} \\
\widetilde{f}(E)=I
\end{array}\right.
$$

where $I$ is the identity mapping of $\tilde{V}$. Define a linear mapping $\widetilde{q}: \widetilde{\mathfrak{g}} \longrightarrow \widetilde{V}$ by

$$
\left\{\begin{array}{l}
\widetilde{q}(Y)=f(Y) v_{0}, \quad Y \in \mathfrak{g}, \\
\widetilde{q}(E)=v_{0} \neq 0 .
\end{array}\right.
$$

Then $\widetilde{q}(\tilde{Y})=\tilde{f}(\tilde{Y}) v_{0}, \tilde{Y} \in \tilde{\mathfrak{g}}$ and we have a real representation $(\tilde{f}, \tilde{V})$ of $\tilde{\mathfrak{g}}$, 
which satisfies that, for $\tilde{X}, \tilde{Y} \in \tilde{\mathfrak{g}}$,

$$
\begin{aligned}
\widetilde{q}([\tilde{X}, \tilde{Y}]) & =\tilde{f}([\tilde{X}, \tilde{Y}]) \widetilde{q}(E) \\
& =[\tilde{f}(\tilde{X}), \widetilde{f}(\tilde{Y})] \widetilde{q}(E) \\
& =\widetilde{f}(\widetilde{X}) \widetilde{f}(\tilde{Y}) \widetilde{q}(E)-\widetilde{f}(\widetilde{Y}) \tilde{f}(\tilde{X}) \widetilde{q}(E) \\
& =\widetilde{f}(\widetilde{X}) \widetilde{q}(\widetilde{Y})-\widetilde{f}(\tilde{Y}) \widetilde{q}(\tilde{X}) .
\end{aligned}
$$

By the condition (\#) and definition of $\widetilde{q}$,

$$
\operatorname{Im}(\widetilde{q})=f(\mathfrak{g}) v_{0} \oplus \mathbf{R} v_{0}=\tilde{V} .
$$

Since $(f, \widetilde{V})$ is spherical, $\operatorname{Ker}(\widetilde{q}) \supset \mathfrak{k}$. Moreover, for the linear mapping $\tilde{q}: \tilde{\mathfrak{g}} \rightarrow \widetilde{V}$

$$
\operatorname{dim} \operatorname{Ker}(\widetilde{q})+\operatorname{dim} \operatorname{Im}(\widetilde{q})=\operatorname{dim} \widetilde{\mathfrak{g}}=\operatorname{dim} \mathfrak{g}+1,
$$

and

$$
\operatorname{dim} \tilde{V}=\operatorname{dim} G / K+1
$$

Thus, we have

$$
\begin{aligned}
\operatorname{dim} \operatorname{Ker}(\widetilde{q}) & =\operatorname{dim} \mathfrak{g}+1-\operatorname{dim} \operatorname{Im}(\widetilde{q}) \\
& =\operatorname{dim} \mathfrak{g}+1-\operatorname{dim} \widetilde{V} \\
& =\operatorname{dim} \mathfrak{k}
\end{aligned}
$$

which implies $\operatorname{Ker}(\widetilde{q})=\mathfrak{k}$. Therefore, $(\tilde{f}, \widetilde{q})$ is the desired one.

Definition 1.7 Let $M=G / K$ be a homogeneous space. A finite dimensional real representation $(f, \widetilde{V})$ of a Lie algebra $\mathfrak{g}$ is called to be admissible if $(f, \tilde{V})$ is spherical relative to $(\mathfrak{g}, \mathfrak{k})$ and $\widetilde{V}$ contains no trivial representation of $\mathfrak{g}$, that is, $\widetilde{V}$ contains no element $v \neq 0$ satisfying that

$$
f(\mathfrak{g}) v=\{0\} .
$$

Proposition 1.8 Let $M=G / K$ be a homogeneous space of dimension $\geq 2$ whose isotropy representation is irreducible, that is, decomposing $\mathfrak{g}$ into sum of $\operatorname{ad}(\mathfrak{k})$ invariant subspaces

$$
\mathfrak{g}=\mathfrak{k} \oplus \mathfrak{m},
$$


and $\mathfrak{m}$ is irreducible under $\operatorname{ad}(\mathfrak{k})$. Then

$$
\begin{aligned}
& \min \{\operatorname{dim} \tilde{V} ;(f, \tilde{V}) \text { is an admissible representation of } \mathfrak{g}\} \\
& \quad \geq \operatorname{dim} M+1 .
\end{aligned}
$$

In particular, if $(f, \tilde{V})$ is an admissible real completely reducible representation of $\mathfrak{g}$ of dimension $\operatorname{dim} M+1,(f, \widetilde{V})$ is irreducible.

Proof. Let $(f, \widetilde{V})$ be an admissible representation of $\mathfrak{g}$. Let $v_{0} \in \tilde{V}$ be a non-zero element of $\widetilde{V}$ satisfying $f(X) v_{0}=0$ for all $X \in \mathfrak{k}$.

Now let $\mathfrak{m}^{\prime}$ be a subspace of $\mathfrak{m}$ defined by

$$
\mathfrak{m}^{\prime}=\left\{X \in \mathfrak{m} ; f(X) v_{0}=0\right\}
$$

Then it is ad( $\mathfrak{k})$ invariant, because

$$
\begin{aligned}
f([X, Y]) v_{0} & =[f(X), f(Y)] v_{0} \\
& =f(X) f(Y) v_{0}-f(Y) f(X) v_{0} \\
& =0,
\end{aligned}
$$

for all $X \in \mathfrak{k}$ and $Y \in \mathfrak{m}^{\prime}$. Since $\mathfrak{m}$ is irreducible under $\operatorname{ad}(\mathfrak{k}), \mathfrak{m}^{\prime}=\{0\}$ or $\mathfrak{m}^{\prime}=\mathfrak{m}$. In the case $\mathfrak{m}^{\prime}=\mathfrak{m}, f(\mathfrak{g}) v_{0}=\{0\}$. Hence $\mathbf{R} v_{0}$ is the trivial representation of $\mathfrak{g}$ which contradicts an admissibility of $(f, \widetilde{V})$. Therefore, $\mathfrak{m}^{\prime}=\{0\}$, i.e.,

$$
\left\{X \in \mathfrak{g} ; f(X) v_{0}=0\right\}=\mathfrak{k} .
$$

Then the mapping $\mathfrak{m} \ni X \mapsto f(X) v_{0} \in \tilde{V}$ is injective and $\operatorname{dim} \mathfrak{m} \leq \operatorname{dim} \tilde{V}$.

Furthermore, we obtain

$$
\operatorname{dim} \mathfrak{m}+1 \leq \operatorname{dim} \tilde{V} .
$$

Otherwise, since

$$
\widetilde{V}=f(\mathfrak{m}) v_{0}
$$

and $v_{0} \in \tilde{V}$, it should hold that, for some $X_{1} \in \mathfrak{m}, v_{0}=f\left(X_{1}\right) v_{0}$. For all $Y \in \mathfrak{k},\left[Y, X_{1}\right] \in \mathfrak{m}$ and

$$
\begin{aligned}
f\left(\left[Y, X_{1}\right]\right) v_{0} & =f(Y) f\left(X_{1}\right) v_{0}-f\left(X_{1}\right) f(Y) v_{0} \\
& =f(Y) v_{0} \\
& =0
\end{aligned}
$$


Therefore, we have

$$
\left[Y, X_{1}\right] \in \mathfrak{k} \cap \mathfrak{m}=\{0\} .
$$

Thus, $\mathbf{R} X_{1} \subset \mathfrak{m}$ is $\operatorname{ad}(\mathfrak{k})$ invariant. By irreducibility of $\mathfrak{m}$ under ad( $(\mathfrak{k})$, it must hold that $\mathfrak{m}=\mathbf{R} X_{1}$, which contradicts that $\operatorname{dim} M \geq 2$.

Proposition 1.9 Let $M=G / K$ be a homogeneous space with $\operatorname{dim} M \geq 2$ whose isotropy representation is irreducible. Let $(f, \tilde{V})$ be a real spherical representation of $(\mathfrak{g}, \mathfrak{k})$, that is, there exists a non-zero element $v_{0}$ in $\widetilde{V}$ satisfying $f(\mathfrak{k}) v_{0}=\{0\}$. If $(f, \widetilde{V})$ is of dimension $\operatorname{dim} M+1$ and satisfies the condition:

$$
\widetilde{V}=f(\mathfrak{g}) v_{0} \oplus \mathbf{R} v_{0}
$$

then $(f, \widetilde{V})$ admits no trivial representation.

Proof. Let

$$
\mathfrak{m}^{\prime}=\left\{X \in \mathfrak{m} ; f(X) v_{0}=0\right\} .
$$

Then by the same way as the proof of Proposition 1.8, we have $\mathfrak{m}^{\prime}=\{0\}$ or $\mathfrak{m}^{\prime}=\mathfrak{m}$. If $\mathfrak{m}^{\prime}=\mathfrak{m}$, then $f(\mathfrak{g}) v_{0}=\{0\}$, which contradicts the condition (\#). We have $\mathfrak{m}^{\prime}=\{0\}$ and then

$$
\mathfrak{k}=\left\{X \in \mathfrak{g} ; f(X) v_{0}=0\right\} .
$$

Now assume that $(f, \tilde{V})$ admits a trivial representation. Then there exists a non-zero element $v \in \widetilde{V}$ satisfying that $f(X) v=0$ for all $X \in \mathfrak{g}$. By the condition (\#), it can be expressed that

$$
v=f\left(X_{1}\right) v_{0}+a v_{0}
$$

for some non-zero element $X_{1} \in \mathfrak{m}$ and real number $a$. We have then

$$
f\left(\left[\mathfrak{k}, X_{1}\right]\right) v_{0}=\{0\} .
$$

Indeed, for all $Y \in \mathfrak{k}$,

$$
\begin{aligned}
f\left(\left[Y, X_{1}\right]\right) v_{0} & =f(Y) f\left(X_{1}\right) v_{0}-f\left(X_{1}\right) f(Y) v_{0} \\
& =f(Y) v \\
& =0 .
\end{aligned}
$$

Therefore, (1.10) and (1.11) imply that $\left[\mathfrak{k}, X_{1}\right] \subset \mathfrak{k}$. Since $\mathfrak{m}$ is invariant 
under $\operatorname{ad}(\mathfrak{k})$, we have

$$
\left[\mathfrak{k}, X_{1}\right] \subset \mathfrak{k} \cap \mathfrak{m}=\{0\} .
$$

In particular, $\mathbf{R} X_{1}$ is an ad(k)-invariant subspace of $\mathfrak{m}$. By means of irreducibility of $\mathfrak{m}$ under $\operatorname{ad}(\mathfrak{k}), \mathfrak{m}$ must be $\mathbf{R} X_{1}$, that is, $\operatorname{dim} M=1$, which contradicts the assumption $\operatorname{dim} M \geq 2$.

By Propositions 1.8 and 1.9, we obtain:

Theorem 1.12 Let $M=G / K$ be a homogeneous space of dimension $\geq 2$ whose isotropy representaton is irreducible. Let $(f, \widetilde{V})$ be a real spherical completely reducible representation of $(\mathfrak{g}, \mathfrak{k})$ of dimension $\operatorname{dim} M+1$ satisfying the condition (\#), that is, there exists a non-zero element in $\widetilde{V}$ such that $f(\mathfrak{k}) v_{0}=\{0\}$ and $\widetilde{V}=f(\mathfrak{g}) v_{0} \oplus \mathbf{R} v_{0}$. Then $(f, \widetilde{V})$ is irreducible.

Furthermore, we obtain:

Theorem 1.13 Assume that $M=G / K$ is an irreducible Riemannian symmetric space of dimension $\geq 2$. Then the irreducible representation $(f, \widetilde{V})$ in Theorem 1.12 is of the first kind, that is, the complexification $\left(f^{\mathbf{C}}, \widetilde{V}^{\mathbf{C}}\right)$ is also irreducible.

Remark 1.14 Theorem 1.13 was obtained by Agaoka in [1, p. 161], for his $(N)$-homomorphism $f$.

Proof. It is well-known that $\mathfrak{m}^{\mathbf{C}}$ is either (i) irreducible or (ii) decomposed into sum of two $\operatorname{ad}\left(\mathfrak{k}^{\mathbf{C}}\right)$-irreducible subspaces $\mathfrak{m}_{ \pm}$of the same dimension. Assume that $\left(f^{\mathbf{C}}, \widetilde{V}^{\mathbf{C}}\right)$ is reducible, i.e., $\widetilde{V}^{\mathbf{C}}$ is decomposed into

$$
\widetilde{V}^{\mathbf{C}}=V_{1} \oplus V_{2}
$$

where $V_{i}$ are non-zero invariant subspaces under $f^{\mathbf{C}}\left(\mathfrak{g}^{\mathbf{C}}\right)$.

In the case (i), we have a decomposition of $\tilde{V}^{\mathbf{C}}$

$$
\widetilde{V}^{\mathbf{C}}=f^{\mathbf{C}}\left(\mathfrak{m}^{\mathbf{C}}\right) v_{0} \oplus \mathbf{C} v_{0},
$$

where $f^{\mathbf{C}}\left(\mathfrak{m}^{\mathbf{C}}\right) v_{0}$ and $\mathbf{C} v_{0}$ are inequivalent, irreducible as $f^{\mathbf{C}}\left(\mathfrak{k}^{\mathbf{C}}\right)$-modules because $\mathfrak{m}^{\mathbf{C}}$ is an irreducible $\operatorname{ad}\left(\mathfrak{k}^{\mathbf{C}}\right)$-module by the assumption. Thus, one of $V_{i}$, say $V_{1}$, must be equal to $f^{\mathbf{C}}\left(\mathfrak{m}^{\mathbf{C}}\right) v_{0}$ and $V_{2}=\mathbf{C} v_{0}$, respectively. But since $V_{2}$ is $f^{\mathbf{C}}\left(\mathfrak{g}^{\mathbf{C}}\right)$-invariant, $\tilde{V}^{\mathbf{C}}$ must satisfy

$$
\tilde{V}^{\mathbf{C}}=f^{\mathbf{C}}\left(\mathfrak{g}^{\mathbf{C}}\right) v_{0} \oplus \mathbf{C} v_{0} \subset V_{2},
$$


which is a contradiction.

In the case (ii), we have two $f^{\mathbf{C}}\left(\mathfrak{k}^{\mathbf{C}}\right)$-invariant decompositions of $\tilde{V}^{\mathbf{C}}$ :

$$
\tilde{V}^{\mathbf{C}}=f^{\mathbf{C}}\left(\mathfrak{m}_{+}\right) v_{0} \oplus f^{\mathbf{C}}\left(\mathfrak{m}_{-}\right) v_{0} \oplus \mathbf{C} v_{0}=V_{1} \oplus V_{2}
$$

Note that the first decomposition of $\widetilde{V}^{\mathbf{C}}$ is an irreducible decomposition as $f^{\mathbf{C}}\left(\mathfrak{k}^{\mathbf{C}}\right)$-modules. Therefore, $V_{1}$ and $V_{2}$ are isomorphic to either $f^{\mathbf{C}}\left(\mathfrak{m}_{+}\right) v_{0}$, $f^{\mathbf{C}}\left(\mathfrak{m}_{-}\right) v_{0} \oplus \mathbf{C} v_{0}$, respectively, or $f^{\mathbf{C}}\left(\mathfrak{m}_{+}\right) v_{0} \oplus f^{\mathbf{C}}\left(\mathfrak{m}_{-}\right) v_{0}, \mathbf{C} v_{0}$, respectively. In any case, $V_{2}$ contains a 1 -dimensional representation of $\mathfrak{g}$, say $U$. Since $\mathfrak{g}$ is semisimple because of irreducibility of $G / K, U$ is a trivial representation. Thus, $\widetilde{V}$ itself, must contain a 1-dimensional trivial representaion, which contradicts the irreducibility of $\widetilde{V}$. We obtain Theorem 1.13.

\section{Affine connections on symmetric spaces}

In this section, we assume $M=G / K$ is an irreducible Riemannian symmetric space of $\operatorname{dim} M \geq 2$. All simply connected irreducible Riemannian symmetric spaces are classified and divided into four types (cf. [4, p. 439]). The compact case is Types (I) and (II). The non-compact case is as follows:

(i) Type (III): These are $(\mathfrak{g}, \mathfrak{k})$, where the Lie algebra $\mathfrak{g}$ is simple and its complexification $\mathfrak{g}^{\mathbf{C}}$ is also simple and $\mathfrak{k}$ is a maximal compact subalgebra of $\mathfrak{g}$. One can see the table of all irreducible symmetric spaces in $[4$, p. 518].

(ii) Type (IV): These are $(\mathfrak{g}, \mathfrak{k})$, where $\mathfrak{g}=\mathcal{G}_{\mathbf{R}}$ is the scalar field restriction to $\mathbf{R}$ of a complex simple Lie algebra $\mathcal{G}$ and $\mathfrak{k}$ is a maximal compact subalgebras of $\mathfrak{g}$. One can see the classification in $[4$, p. 516].

Now we give examples of Riemannian symmetric spaces admitting the desired representation $(f, \tilde{V})$. Examples 2, 3, 4 and 6 are of type (III) and Examples 1, 2, 3, and 4 have been already known (cf. [1] or [13]) and Example 6 is new and Example 5 is of type (IV) and quite new.

Example 1 (cf. [1], [13]). $S O(n+1) / S O(n) . \quad$ In this case,

$$
\begin{aligned}
\mathfrak{g} & =\mathfrak{o}(n+1)=\left\{X \in \mathfrak{g l}(n+1, \mathbf{R}) ; X+{ }^{t} X=0\right\}, \\
\mathfrak{k} & =\left\{\left(\begin{array}{cc}
0 & 0 \\
0 & A
\end{array}\right) ; A \in \mathfrak{o}(n)\right\}, \\
\mathfrak{m} & =\left\{\left(\begin{array}{cc}
0 & -{ }^{t} a \\
a & O
\end{array}\right) ; a \in \mathbf{R}^{n}\right\} .
\end{aligned}
$$


Let us consider a real representation of $\mathfrak{g},(f, \tilde{V})$ given by $\tilde{V}=\mathbf{R}^{n+1}$, the space of $n+1$-column real vectors, and

$$
f(X) u=X u, \quad X \in \mathfrak{g}, \quad \in \mathbf{R}^{n+1} .
$$

Let $v_{0}={ }^{t}(1,0, \ldots, 0) \in \mathbf{R}^{n+1}$. Then this representation $(f, \tilde{V})$ satisfies $\left\{X \in \mathfrak{g} ; f(X) v_{0}=0\right\}=\mathfrak{k}, \widetilde{V}=f(\mathfrak{g}) v_{0} \oplus \mathbf{R} v_{0}$, and $\operatorname{dim} \widetilde{V}=\operatorname{dim} \mathfrak{m}+1=$ $n+1$.

Example 2 (cf. [1], [13]). $S L(n+1, \mathbf{R}) / S O(n+1)$. Let

$$
\begin{aligned}
\mathfrak{g} & =\mathfrak{s l}(n+1, \mathbf{R})=\{X \in \mathfrak{g l}(n+1, \mathbf{R}) ; \operatorname{Tr}(X)=0\}, \\
\mathfrak{k} & =\mathfrak{o}(n+1), \quad \mathfrak{m}=\left\{A \in \mathfrak{g} ;{ }^{t} A=A\right\} .
\end{aligned}
$$

Let us consider an $\frac{1}{2}(n+1)(n+2)$-dimensional representation $(f, \tilde{V})$ of $\mathfrak{g}$ given by

$$
\begin{aligned}
& \tilde{V}=\left\{A \in \mathfrak{g l}(n+1, \mathbf{R}) ;{ }^{t} A=A\right\}, \\
& f(X) A=X A+A^{t} X, \quad X \in \mathfrak{g}, \quad A \in \widetilde{V} .
\end{aligned}
$$

Let $v_{0}=I \in \tilde{V}$ be the identity matrix. Then we have

$$
\left\{X \in \mathfrak{g} ; f(X) v_{0}=0\right\}=\mathfrak{k}, \quad \widetilde{V}=f(\mathfrak{g}) v_{0} \oplus \mathbf{R} v_{0},
$$

and $\operatorname{dim} \tilde{V}=\operatorname{dim} \mathfrak{m}+1=\frac{1}{2}(n+1)(n+2)$.

Example 3 (cf. [1]). $S U^{*}(2 n) / S p(n)$. Let

$$
\begin{aligned}
\mathfrak{g} & =\mathfrak{s u}^{*}(2 n)=\mathfrak{s l}(n, \mathbf{H})=\left\{X \in \mathfrak{s l}(2 n, \mathbf{C}) ; J_{n} \bar{X}=X J_{n}\right\} \\
& =\left\{\left(\begin{array}{cc}
A & B \\
-\bar{B} & \bar{A}
\end{array}\right) ; A, B \in \mathfrak{g l}(n, \mathbf{C}), \operatorname{Tr}(A+\bar{A})=0\right\} .
\end{aligned}
$$

Then we have

$$
\mathfrak{g}=\mathfrak{k} \oplus \mathfrak{m},
$$

where

$$
\begin{aligned}
\mathfrak{k} & =\left\{X \in \mathfrak{g} ;{ }^{t} \bar{X}=-X\right\}=\left\{\left(\begin{array}{cc}
A & B \\
-\bar{B} & \bar{A}
\end{array}\right) ;{ }^{t} \bar{A}=-A,{ }^{t} B=B\right\}, \\
\mathfrak{m} & =\left\{X \in \mathfrak{g} ;{ }^{t} \bar{X}=X\right\}
\end{aligned}
$$




$$
=\left\{\left(\begin{array}{cc}
A & B \\
-\bar{B} & \bar{A}
\end{array}\right) ;{ }^{t} \bar{A}=A, \operatorname{Tr}(A)=0,{ }^{t} B=-B\right\} .
$$

Here $J_{n}$ is the matrix given by

$$
J_{n}=\left(\begin{array}{cc}
O & -I_{n} \\
I_{n} & O
\end{array}\right)
$$

where $I_{n}$ is the identity matrix of degree $n$. Let us define a mapping $J$ of $\bigwedge^{2} \mathbf{C}^{2 n}$ into itself by

$$
J\left(w_{1} \wedge w_{2}\right)=J w_{1} \wedge J w_{2}, \quad J(w)=J_{n} \bar{w}, \quad w_{1}, w_{2}, w \in \mathbf{C}^{2 n}
$$

Define $\widetilde{V}$ by

$$
\begin{aligned}
\tilde{V}= & \left\{v \in \bigwedge^{2} \mathbf{C}^{2 n} ; J v=v\right\} \\
= & \left\{v=\sum_{i, j=1}^{n}\left(a_{i j} e_{i} \wedge e_{n+j}+b_{i j} e_{i} \wedge e_{j}+\bar{b}_{i j} e_{n+i} \wedge e_{n+j}\right) ;\right. \\
& \left.a_{i j}=\bar{a}_{j i}, b_{i j}+b_{j i}=0, a_{i j}, b_{i j} \in \mathbf{C}\right\},
\end{aligned}
$$

where $e_{i}, 1 \leq i \leq 2 n$, is the standard basis of $\mathbf{C}^{2 n}$. The space $\tilde{V}$ is a real $\left(\begin{array}{c}2 n \\ 2\end{array}\right)=n(2 n-1)$ dimensional space and $\mathfrak{g}$ acts on it naturally. Let us take

$$
v_{0}=e_{1} \wedge e_{n+1}+e_{2} \wedge e_{n+2}+\cdots+e_{n} \wedge e_{2 n},
$$

and for $X \in \mathfrak{g}$,

$$
\begin{aligned}
f(X) v_{0} & =X v_{0} \\
& =\sum_{i, j=1}^{n}\left\{\left(A_{i j}+\bar{A}_{j i}\right) e_{i} \wedge e_{n+j}+B_{j i} e_{i} \wedge e_{j}-\bar{B}_{i j} e_{n+i} \wedge e_{n+j}\right\} .
\end{aligned}
$$

Thus, we have

$$
\left\{X \in \mathfrak{g} ; f(X) v_{0}=0\right\}=\mathfrak{k}, \quad \tilde{V}=f(\mathfrak{g}) v_{0} \oplus \mathbf{R} v_{0},
$$

and $\operatorname{dim} \widetilde{V}=\operatorname{dim} \mathfrak{m}+1=n(2 n-1)$.

Example 4 (cf. [1], [13]). $S O_{o}(n, 1) / S O(n)$. Let

$$
\mathfrak{g}=\mathfrak{o}(n, 1)=\left\{X \in \mathfrak{g l}(n+1, \mathbf{R}) ;{ }^{t} X J^{\prime}+J^{\prime} X=0\right\},
$$


where $J^{\prime}$ is the matrix of degree $n+1$ given by

$$
J^{\prime}=\left(\begin{array}{cc}
-1 & 0 \\
0 & I_{n}
\end{array}\right)
$$

Then we have

$$
\mathfrak{g}=\mathfrak{k} \oplus \mathfrak{m},
$$

where

$$
\begin{aligned}
\mathfrak{k} & =\mathfrak{o}(n)=\left\{\left(\begin{array}{cc}
0 & 0 \\
0 & A
\end{array}\right) ; A \in \mathfrak{o}(n)\right\}, \\
\mathfrak{m} & =\left\{\left(\begin{array}{cc}
0 & { }^{t} a \\
a & O
\end{array}\right) ; a \in \mathbf{R}^{n}\right\} .
\end{aligned}
$$

Let us consider a real representation $(f, \tilde{V})$ of $\mathfrak{g}$ given by $\widetilde{V}=\mathbf{R}^{n+1}$ and

$$
f(X) u=X u, \quad X \in \mathfrak{g}, \quad u \in \mathbf{R}^{n+1} .
$$

Let us take $v_{0}={ }^{t}(1,0, \ldots, 0) \in \mathbf{R}^{n+1}$. Then we have

$$
\left\{X \in \mathfrak{g} ; f(X) v_{0}=0\right\}=\mathfrak{k}, \widetilde{V}=f(\mathfrak{g}) v_{0} \oplus \mathbf{R} v_{0},
$$

and $\operatorname{dim} \tilde{V}=\operatorname{dim} \mathfrak{m}+1=n+1$.

Example 5. $S L(n+1, \mathbf{C}) / S U(n+1)$. Let

$$
\mathfrak{g}=\mathcal{G}_{\mathbf{R}}=\mathfrak{s l}(n+1, \mathbf{C})=\{X \in \mathfrak{g l}(n+1, \mathbf{C}) ; \operatorname{Tr}(X)=0\} .
$$

Then we have

$$
\mathfrak{g}=\mathfrak{k} \oplus \mathfrak{m},
$$

where

$$
\begin{aligned}
\mathfrak{k} & =\mathfrak{s u}(n+1)=\left\{A \in \mathfrak{g l}(n+1, \mathbf{C}) ;{ }^{t} \bar{A}=-A, \operatorname{Tr}(A)=0\right\}, \\
\mathfrak{m} & =\left\{A \in \mathfrak{g l}(n+1, \mathbf{C}) ;{ }^{t} \bar{A}=A, \operatorname{Tr}(A)=0\right\},
\end{aligned}
$$

and $\operatorname{dim} \mathfrak{m}=(n+1)^{2}-1=n^{2}+2 n$. Let us consider a real representation $(f, \widetilde{V})$ of $\mathfrak{g}$ given by

$$
\widetilde{V}=\left\{A \in \mathfrak{g l}(n+1, \mathbf{C}) ;{ }^{t} \bar{A}=A\right\},
$$

which is the space of hermitian matrices of degree $n+1$, is of real dimension 
$(n+1)^{2}$. The action of $\mathfrak{g}$ on it is given by

$$
f(X) A=X A+A^{t} \bar{X}, \quad X \in \mathfrak{g}, \quad A \in \tilde{V} .
$$

Let us take $v_{0}=I_{n+1} \in \widetilde{V}$ the identity matrix of degree $n+1$. Then we obtain

$$
\left\{X \in \mathfrak{g} ; f(X) v_{0}=0\right\}=\mathfrak{k}, \widetilde{V}=f(\mathfrak{g}) v_{0} \oplus \mathbf{R} v_{0},
$$

and $\operatorname{dim} \tilde{V}=\operatorname{dim} \mathfrak{m}+1=(n+1)^{2}$.

Example 6. $\quad E_{6} / F_{4}$ (of non-compact type). This is the EIV type symmetric space of non-compact type. By [10, p. 298], there exists a complex irreducible representation $\left(f, V_{\lambda_{6}}\right)$ of $\mathfrak{g}^{\mathbf{C}}$ of complex dimension 27 such that, considered as an $f\left(\mathfrak{k}^{\mathrm{C}}\right)$-module,

$$
V_{\lambda_{6}}=W \oplus \mathbf{C} w
$$

where $W$ is a complex irreducible representation of $\mathfrak{k}^{\mathbf{C}}$ and $f\left(\mathfrak{k}^{\mathbf{C}}\right) w=\{0\}$. Here $\lambda_{i}(i=1, \ldots, 6)$ are the fundamental weights corresponding to the fundamental roots $\left\{\alpha_{i}\right\}(i=1, \ldots, 6)$ of $\mathfrak{g}^{\mathbf{C}}$. By the table in [14, p. 14], and Theorem 10.4 in $\left[14\right.$, p. 113], it turns out that $\left(f, V_{\lambda_{6}}\right)$ is of the first kind, that is, there exists a real irreducible representation $(f, \widetilde{V})$ of $\mathfrak{g}$ of dimension 27 such that $\widetilde{V}^{\mathbf{C}}=V_{\lambda_{6}}$. This representation $(f, \widetilde{V})$ is the desired one.

Indeed, putting $w=v_{0}+\sqrt{-1} v_{0}^{\prime}$, where $v_{0}, v_{0}^{\prime} \in \widetilde{V}$, we may assume $v_{0} \neq 0$ taking $\sqrt{-1} w$ instead if necessary. We have

$$
f(\mathfrak{k}) v_{0}=\{0\} \quad \text { and } \quad \mathbf{C} v_{0}=\mathbf{C} w .
$$

In fact, if $\mathbf{C} v_{0} \neq \mathbf{C} w, \mathbf{C} v_{0}+\mathbf{C} w$ is a 2 dimensional trivial $f\left(\mathfrak{k}^{\mathbf{C}}\right)$-module of $V_{\lambda_{6}}$, which contradicts the condition $(*)$. Since $f\left(\mathfrak{g}^{\mathbf{C}}\right) v_{0}$ is a non-zero $f\left(k^{\mathbf{C}}\right)$-module and since $W$ and $\mathbf{C} v_{0}$ are irreducible as $f\left(\mathfrak{k}^{\mathbf{C}}\right)$-modules and inequivalent to each other, $f\left(\mathfrak{g}^{\mathbf{C}}\right) v_{0}$ must be one of $\widetilde{V}^{\mathbf{C}}, W$ or $\mathbf{C} v_{0}$.

Here the kernel of the mapping $\Phi: \mathfrak{g}^{\mathbf{C}} \ni X \mapsto f(X) v_{0} \in \widetilde{V}^{\mathbf{C}}$ contains $\mathfrak{k}^{\mathbf{C}}$. Thus, we have

$$
\operatorname{dim} \operatorname{Im}(\Phi) \leq \operatorname{dim} \mathfrak{g}^{\mathbf{C}}-\operatorname{dim} \mathfrak{k}^{\mathbf{C}}=\operatorname{dim} M=26 .
$$

Therefore, $\Phi$ is not surjective, that is, $f\left(\mathfrak{g}^{\mathbf{C}}\right) v_{0} \neq \widetilde{V}^{\mathbf{C}}$. Moreover, $f\left(\mathfrak{g}^{\mathbf{C}}\right) v_{0} \neq$ $\mathbf{C} v_{0}$. Because, if so, $f\left(\mathfrak{g}^{\mathbf{C}}\right) v_{0}$ is an $f\left(\mathfrak{g}^{\mathbf{C}}\right)$-invariant subspace of $\widetilde{V}^{\mathbf{C}}$, which contradicts the irreducibility of $\widetilde{V}^{\mathbf{C}}$. Thus, we obtain $f\left(\mathfrak{g}^{\mathbf{C}}\right) v_{0}=W$ and

$$
\tilde{V}^{\mathbf{C}}=f\left(\mathfrak{g}^{\mathbf{C}}\right) v_{0} \oplus \mathbf{C} v_{0},
$$


which implies $\operatorname{dim}_{\mathbf{C}} f\left(\mathfrak{g}^{\mathbf{C}}\right) v_{0}=26$. Moreover, since $\operatorname{Ker}(\Phi) \supset \mathfrak{k}^{\mathbf{C}}$ and $\operatorname{dim}_{\mathbf{C}} f\left(\mathfrak{g}^{\mathbf{C}}\right) v_{0}=26$, we have $\operatorname{Ker}(\Phi)=\mathfrak{k}^{\mathbf{C}}$ and then the kernel of the restriction of $\Phi$ to $\mathfrak{g}$ is equal to $\mathfrak{k}$. Therefore, $\operatorname{dim}_{\mathbf{R}} f(\mathfrak{g}) v_{0}=26=\operatorname{dim} M$ and

$$
\widetilde{V}=f(\mathfrak{g}) v_{0} \oplus \mathbf{R} v_{0}
$$

Lemma 2.1 Let $M=G / K$ be a compact simply connected homogeneous space admitting a real representation $(f, \widetilde{V})$ of $\mathfrak{g}$ of dimension $\operatorname{dim} M+1$, which is spherical relative to $(\mathfrak{g}, \mathfrak{k})$ and satisfies

$$
\widetilde{V}=f(\mathfrak{g}) v_{0} \oplus \mathbf{R} v_{0} .
$$

Then $M$ is a unit sphere.

Proof. We may assume $G$ is a simply connected compact Lie group and $K$ is a closed subgroup. Since $G$ is compact, $\widetilde{V}$ admits an $f(G)$-invariant inner product (, ) in such a way $\left\|v_{0}\right\|=1$. Then $f(G) v_{0} \subset S^{n}=\{v \in \widetilde{V} ;\|v\|=$ $1\}$, where $n=\operatorname{dim} M$. By the assumption $\operatorname{dim} M=\operatorname{dim} \tilde{V}-1=n, f(G) v_{0}$ is an open and closed subset in $S^{n}$. Therefore, $M=f(G) v_{0}=S^{n}$.

Main result in this section is the following:

Theorem 2.2 Let $M=G / K$ be an irreducible simply connected Riemannian symmetric space. Then $M=G / K$ admits a $G$-invariant projectively flat affine connection if and only if $M=G / K$ is one of the following:
(1) $S O(n+1) / S O(n), n \geq 2$,
(2) $S L(n+1, \mathbf{R}) / S O(n+1), n \geq 2$,
(3) $S U^{*}(2 n) / S p(n), n \geq 3$,
(4) $S O_{o}(n, 1) / S O(n), n \geq 2$,
(5) $S L(n+1, \mathbf{C}) / S U(n+1), n \geq 1$,
(6) $E_{6} / F_{4}$ (non-compact type of EIV).

Proof. By Lemma 2.1, we may assume $M$ is non-compact. Due to Theorem 1.13 , we only have to find a complex irreducible representation $(\rho, W)$ of $\mathfrak{g}$ of the first kind, i.e., $W$ is the complexification of a real irreducible one $\widetilde{V}$, and of complex dimension $\operatorname{dim}_{\mathbf{C}} W=\operatorname{dim} M+1$.

Type (III). Let $(\mathfrak{g}, \mathfrak{k})$ be a pair of real simple Lie algebras such that the complexification $\mathfrak{g}^{\mathbf{C}}$ is simple, and $\mathfrak{k}$ is a maximal compact Lie subalgebra. In the exceptional case, we can see a list of dimensions of $M=G / K$ 
in $[4$, p. 518], and a list of the complex irreducible representation of the exceptional Lie algebras $\mathfrak{g}^{\mathbf{C}}$ of dimension $\leq \operatorname{dim} M+1$ in [10]. It turns out that the only exceptional symmetric space $M=G / K$ of type (III) admitting the complex irreducible represenatations $(\rho, W)$ of $\mathfrak{g}^{\mathbf{C}}$ of dimension $\operatorname{dim}_{\mathbf{C}} W=\operatorname{dim} M+1$ is non-compact type of EIV, $M=E_{6} / F_{4}$ $(\operatorname{dim} M=26)$. But this is the case, Example 6. Together with Agaoka's classification (cf. [1]) of all classical symmetric spaces of type (III) admitting the desired representations, we have accomplished the classification in case of type (III).

Type (IV). This case is a pair $(\mathfrak{g}, \mathfrak{k})$, where $\mathfrak{g}$ is the restriction to the real field of a complex simple Lie algebra $\mathcal{G}$ and $\mathfrak{k}$ is a maximal compact Lie subalgebra of $\mathfrak{g}$. In this case, it is known (cf. [3] or [14]) that every irreducible real representation of the first kind of $\mathfrak{g}$ is realized as $(\widetilde{\rho}, H(W))$, where

$$
\begin{aligned}
& H(W)=\left\{A \in M_{n}(\mathbf{C}) ;{ }^{t} \bar{A}=A\right\}, \\
& \widetilde{\rho}(X) A=\rho(X) A+A^{t} \overline{\rho(X)}, \quad X \in \mathfrak{g}, \quad A \in H(W),
\end{aligned}
$$

for a complex irreducible representation $(\rho, W)$ of the complex Lie algebra $\mathcal{G}$. The real dimension $\operatorname{dim} H(W)$ is $n^{2}$, where $n=\operatorname{dim}_{\mathbf{C}} W$. This condition excludes the exceptional cases for a complex Lie algebra $\mathcal{G}$. Furthermore, if $\mathcal{G}$ is one of $\mathfrak{o}(2 m+1, \mathbf{C})(m \geq 3), \mathfrak{s p}(m, \mathbf{C})(m \geq 3)$, or $\mathfrak{o}(2 m, \mathbf{C})(m \geq 4)$, the dimension $n=\operatorname{dim}_{\mathbf{C}} W$ satisfying the above condition

$$
n^{2}-1=\operatorname{dim} M=\operatorname{dim} G / K
$$

is smaller than $2 m$. However, the least dimension of a non-trivial finite complex representation of the above $\mathcal{G}$ is $2 m+1,2 m$, or $2 m$, respectively (cf. $[10$, p. 4, p. 52]). These cases do not occur. The case $\mathfrak{o}(5, \mathbf{C})$ also does not occur, because in this case, $\operatorname{dim} M+1=11$ which can not be expressed as $n^{2}$ for some integer $n$. Therefore, one can see the only case which $\operatorname{dim} H(W)=\operatorname{dim} M+1$ holds is that (5) $S L(n+1, \mathbf{C}) / S U(n+1)$ and the desired representation is

$$
\widetilde{V}=\left\{A \in \mathfrak{g l}(n+1, \mathbf{C}) ;{ }^{t} \bar{A}=A\right\},
$$

for the complex representation of $\mathfrak{s l}(n+1, \mathbf{C}), W=\mathbf{C}^{n+1}$. Therefore, we have Theorem 2.2. 


\section{Left invariant affine connections on simple Lie groups}

In this section, we classify all left invariant projectively flat affine connections on simple Lie groups. Existence of such connections on simply connected Lie groups $G$ is equivalent to the existence of a real representation $(f, \widetilde{V})$ of the Lie algebra $\mathfrak{g}$ of dimension $\operatorname{dim} \mathfrak{g}+1$ such that

$$
\tilde{V}=f(\mathfrak{g}) v_{0} \oplus \mathbf{R} v_{0}
$$

for some non-zero element $v_{0} \in \tilde{V}$.

Our main theorem is

Theorem 3.1 Let $G$ be a real simple Lie group. If $G$ admits a left invariant projectively flat affine connection, then $\mathfrak{g}$ is one of the following:

(a) $\mathfrak{o}(3) \cong \mathfrak{s u}(2)$

(b) $\mathfrak{s l}(n+1, \mathbf{R}), n \geq 1$,

(c) $\mathfrak{s u}^{*}(2 n), n \geq 2$,

(d) $\mathfrak{s u}(r, s)(r+s=$ even, $r+s \geq 4) ; \mathfrak{o}(3,4) ; \mathfrak{o}(1,9), \mathfrak{o}(5,5) ; \mathfrak{o}(3,11)$, $\mathfrak{o}(7,7)$.

To show Theorem 3.1, we need the following lemma which was obtained by Agaoka [1]:

Lemma 3.2 Assume that $(f, \widetilde{V})$ is a real representation of a real semisimple Lie algebra $\mathfrak{g}$ of dimension $\operatorname{dim} \mathfrak{g}+1$ satisfying the condition (\#) :

$$
\widetilde{V}=f(\mathfrak{g}) v_{0} \oplus \mathbf{R} v_{0}
$$

for some non-zero element $v_{0} \in \tilde{V}$. Then $(f, \tilde{V})$ contains no trivial representation of $\mathfrak{g}$.

Outline of Proof. For completeness, we give a proof of Lemma 3.2. Let $(f, \widetilde{V})$ be a real representation of a real semisimple Lie algebra $\mathfrak{g}$ satisfying the condition (\#) and of dimension $\operatorname{dim} \mathfrak{g}+1$. By theorem 1.3, there exists a left invariant projectively flat connection $D$ on $G$ with no torsion. Assume that $(f, \widetilde{V})$ contains a trivial representation of $\mathfrak{g}$. Since $\mathfrak{g}$ is semisimple, there exists an $f(\mathfrak{g})$-submodule $W$ and a nonzero vector $v$ of $\widetilde{V}$ satisfying that

$$
\widetilde{V}=W \oplus \mathbf{R} v \quad \text { and } \quad f(\mathfrak{g}) v=\{0\} .
$$

Agaoka (cf. Theorem 3.7 in [1]) showed that $D$ is affinely flat due to this 
condition. However, by a non-existence theorem of left invariant flat affine connection on a real semisimple Lie group due to Matsushima-Okamoto (cf. [9]), we obtain Lemma 3.2.

Now all real simple Lie algebras are classified as follows:

(I) compact simple Lie algebras $\mathfrak{g}$,

(II) $\mathfrak{g}=\mathcal{G}_{\mathbf{R}}$, where $\mathcal{G}$ is a complex simple Lie algebra,

(III) a non-compact real simple Lie algebra $\mathfrak{g}$ of which complexification $\mathfrak{g}^{\mathbf{C}}$ is simple.

For the case (I), the only compact simple Lie algebra admitting a left invariant projectively flat connection is $\mathfrak{s u}(2) \cong \mathfrak{o}(3)$ due to Lemma 2.1.

For the case (II), we have

Lemma 3.3 There is no real simple Lie algebra of type (II) which admits left invariant projectively flat affine connections.

Proof. Let $\mathfrak{g}$ be a real simple Lie algebra of type (II). It is known (cf. [3], [14]) that all real irreducible representations of $\mathfrak{g}$ are exhausted by

(i) $\left(\rho_{\Lambda}, H(V)\right)$, where

$$
H(V):=\left\{A \in M_{n}(\mathbf{C}) ;{ }^{t} \bar{A}=A\right\}, \quad \rho_{\Lambda}(X) A:=\rho(X) A+A^{t} \overline{\rho(X)}
$$

for $X \in \mathfrak{g}=\mathcal{G}_{\mathbf{R}}, A \in H(V)$, and

(ii) $\left(\rho_{\mathbf{R}}, V_{\mathbf{R}}\right)$, the representation restricted to the real field of $(\rho, V)$, for a complex irreducible representation $(\rho, V)$ of $\mathcal{G}$.

Note that $\operatorname{dim} H(V)=n^{2}$, and $\operatorname{dim} V_{\mathbf{R}}=2 n$, where $n=\operatorname{dim}_{\mathbf{C}} V$.

On the other hand, all complex simple Lie algebras $\mathcal{G}$ are classified in [4]. By Lemma 3.2, $\widetilde{V}$ must be the direct sum of a finite number of representations of the above (i) or (ii), say $\widetilde{V}=\sum H\left(V_{i}\right) \oplus \sum V_{j}^{\prime}{ }_{\mathbf{R}}$, with $\operatorname{dim} H\left(V_{i}\right)>1$. Then it holds that

$$
\begin{aligned}
2 \operatorname{dim}_{\mathbf{C}} \mathcal{G}+1 & =\operatorname{dim} \tilde{V} \\
& =\sum n_{i}{ }^{2}+2 \sum n_{j}{ }^{\prime},
\end{aligned}
$$

where $n_{i}=\operatorname{dim} V_{i}$ and $n_{j}{ }^{\prime}=\operatorname{dim} V_{j}{ }^{\prime} \geq 2$. Comparing the dimensions of complex irreducible representations of $\mathcal{G}$ which may appear in the above equation and making use of Weyl's dimension formula for the classical cases and the tables in [10] for the exceptional cases, it turns out there is no representation $\widetilde{V}$ satisfying the above equality. For example, if $\mathcal{G}=\mathfrak{s l}(n+$ $1, \mathbf{C}),(n \geq 1)$, the possible dimensions of irreducible representations are 
$n+1, \frac{1}{2} n(n+1), \frac{1}{2}(n+1)(n+2)$. But there exists no integral solution $x$, $y, z$ and $w$ satisfying that

$$
\begin{aligned}
& (n+1)^{2} x+2(n+1) y+n(n+1) z+(n+1)(n+2) w \\
& \quad=2\left(n^{2}+2 n\right)+1
\end{aligned}
$$

since the right hand side can not be divided by $n+1$. The similar hold for the remains.

To prove Theorem 3.1 for the case of a real simple Lie algebra $\mathfrak{g}$ of type (III), let us recall representation theory of $\mathfrak{g}$. Classification of real simple Lie algebras $\mathfrak{g}$ of type (III) is by Satake diagram and classification of real irreducible representations of $\mathfrak{g}$ is given in [3], [5] and [14].

Let $\Pi$ be a $\sigma$ fundamental system corresponding to a real simple Lie algebra $\mathfrak{g}$ whose complexification $\mathfrak{g}^{\mathbf{C}}$ is simple. Let $\mathcal{C}(\mathfrak{g})$ be the set of equivalence classes of complex irreducible representations of $\mathfrak{g}$ (also $\mathfrak{g}^{\mathbf{C}}$ ). Then $\mathcal{C}(\mathfrak{g})$ corresponds bijectively to the set $D$ of dominant integral forms of $\mathfrak{g}^{\mathbf{C}}$ relative to $\Pi$. Namely, the correspondence $D \ni \lambda \mapsto\left(\rho_{\lambda}, V_{\lambda}\right) \in \mathcal{C}(\mathfrak{g})$ is bijective, where $\left(\rho_{\lambda}, V_{\lambda}\right) \in \mathcal{C}(\mathfrak{g})$ is the complex irreducible representation of $\mathfrak{g}$ whose highest weight is $\lambda$.

Two complex irreducible representations $\rho$ and $\sigma$ are said to be $\approx-$ equivalent, denoted by $\rho \approx \sigma$, if the complex conjugation $\bar{\rho}$ or $\rho$ itself, is equivalent to $\sigma$. Let $\widehat{\mathcal{C}}(\mathfrak{g})$ be the set of $\approx$-equivalence classes of complex irreducible representations of $\mathfrak{g}$. A complex irreducible representation $(\rho, V)$ is of the first kind (resp. second kind) if $\left(\rho_{\mathbf{R}}, V_{\mathbf{R}}\right)$ is reducible (resp. irreducible). A real irreducible $(r, E)$ is of the first kind (resp. second kind) if the complexification $\left(r^{\mathbf{C}}, E^{\mathbf{C}}\right)$ is irreducible (resp. reducible). Let us denote by $\mathcal{R}(\mathfrak{g})$ the set of equivalence classes of real irreducible representations of $\mathfrak{g}$. Let us denote by $\mathcal{R}^{\mathrm{I}}(\mathfrak{g})$ the set of elements in $\mathcal{R}(\mathfrak{g})$ of the first kind, $\mathcal{R}^{\mathrm{II}}(\mathfrak{g})$ the one of the second kind, $\mathcal{C}^{\mathrm{I}}(\mathfrak{g})$ the set of elements in $\mathcal{C}(\mathfrak{g})$ of the first kind, $\mathcal{C}^{\mathrm{II}}(\mathfrak{g})$, the one of the second kind, and $\widehat{\mathcal{C}}^{\mathrm{II}}(\mathfrak{g})$, the $\approx$-equivalence classes of complex representations of the second kind, respectively. Cartan's fundamental theorem says that

Theorem 3.4 (1) We have the disjoint unions:

$$
\mathcal{R}(\mathfrak{g})=\mathcal{R}^{\mathrm{I}}(\mathfrak{g}) \cup \mathcal{R}^{\mathrm{II}}(\mathfrak{g}), \quad \mathcal{C}(\mathfrak{g})=\mathcal{C}^{\mathrm{I}}(\mathfrak{g}) \cup \mathcal{C}^{\mathrm{II}}(\mathfrak{g}) .
$$

(2) The coefficient field extension induces a bijection of $\mathcal{R}^{\mathrm{I}}(\mathfrak{g})$ onto $\mathcal{C}^{\mathrm{I}}(\mathfrak{g})$. 
(3) The coefficient field restriction induces a bijection of $\widehat{\mathcal{C}}^{\mathrm{II}}(\mathfrak{g})$ onto $\mathcal{R}^{\mathrm{II}}(\mathfrak{g})$.

We show Theorem 3.1 by a case by case checking.

We first show two examples of real simple Lie groups admitting left invariant projectively flat affine connections:

Example 7 (cf. [1]). Let $\mathfrak{g}=\mathfrak{s l}(n+1, \mathbf{R})$ be of type $A I_{n}(n \geq 1), G=$ $S L(n+1, \mathbf{R})$. Let us consider a real representation $\widetilde{V}=\mathfrak{g l}(n+1, \mathbf{R})$ of dimension $(n+1)^{2}$. The Lie algebra $\mathfrak{g}=\mathfrak{s l}(n+1, \mathbf{R})$ acts on it by

$$
f(X) Y:=X Y, \quad X \in \mathfrak{g}, Y \in \mathfrak{g l}(n+1, \mathbf{R}) .
$$

Taking $v_{0}=I$, the identity matrix in $\mathfrak{g l}(n+1, \mathbf{R})$, we have $\widetilde{V}=f(\mathfrak{g}) v_{0} \oplus \mathbf{R} v_{0}$. Since $\operatorname{dim} \widetilde{V}=\operatorname{dim} \mathfrak{g}+1$, this representation satisfies all the conditions of Theorem 1.3. Note that $\widetilde{V}=\mathfrak{g l}(n+1, \mathbf{R})=\mathbf{R}^{n+1} \oplus \cdots \oplus \mathbf{R}^{n+1}$, which is reducible.

Example 8 (cf. [1] ). Let $\mathfrak{g}=\mathfrak{s u}^{*}(2 n)$ be a simple Lie algebra of type $A I I_{n}$ $(n \geq 2)$ of dimension $4 n^{2}-1$ given by

$$
\mathfrak{g}=\left\{\left(\begin{array}{cc}
A & B \\
-\bar{B} & \bar{A}
\end{array}\right) ; A, B \in \mathfrak{g l}(n, \mathbf{C}), \operatorname{Tr}(A+\bar{A})=0\right\} .
$$

Let us consider the following representation $(f, \widetilde{V})$ of dimension $4 n^{2}$ :

$$
\tilde{V}=\left\{\left(\begin{array}{cc}
A & B \\
-\bar{B} & \bar{A}
\end{array}\right) ; A, B \in \mathfrak{g l}(n, \mathbf{C})\right\},
$$

and the action of $\mathfrak{g}$ on it is

$$
f(X) Y:=X Y, \quad X \in \mathfrak{g}, Y \in \tilde{V} .
$$

Taking

$$
v_{0}=\left(\begin{array}{cc}
I & O \\
O & I
\end{array}\right)
$$

it turns out that $(f, \widetilde{V})$ satisfies the conditions of Theorem 1.3 and $S U^{*}(2 n)$ admits a left invariant projectively flat affine connection.

Now we have to show all the other simple Lie groups have no left invariant projectively flat affine connections except the case (d) in Theorem 3.1. 
In the following, we denote by $\rho_{\varpi_{i}}$ the representation in $\mathcal{C}(\mathfrak{g})$ of which highest weight is the fundamental weight $\varpi_{i}$ corresponding to the fundamental roots $\alpha_{i} \in \Pi$. Numbering of $\Pi$ will be followed from [2] and [14].

We show

Lemma 3.5 Let $\mathfrak{g}$ be a real simple Lie algebra of exceptional type with simple $\mathfrak{g}^{\mathbf{C}}$. Then $G$ admits no left invariant projectively flat affine connection.

Proof. Let $\mathfrak{g}$ be a real simple Lie algebra in Lemma 3.5. We first determine low dimensional real representations of $\mathfrak{g}$. By Theorem 3.4, we only have to determine which complex irreducible representations $\left(\rho_{\lambda}, V_{\lambda}\right)$ are of the first kind or the second kind using Cartan's criterion (cf. [14]):

$$
\begin{array}{cl}
\mathfrak{g}=\mathrm{EI} & \operatorname{dim} \mathfrak{g}=78, \quad \rho_{\varpi_{1}}, \rho_{\varpi_{6}} \quad \text { first kind, } \\
& \operatorname{dim}_{\mathbf{C}} \rho_{\varpi_{1}}=\operatorname{dim}_{\mathbf{C}} \rho_{\varpi_{6}}=27,
\end{array}
$$

EII $\operatorname{dim} \mathfrak{g}=78, \quad \rho_{\varpi_{1}}, \rho_{\varpi_{6}} \quad$ second kind,

$$
\operatorname{dim}_{\mathbf{C}} \rho_{\varpi_{1}}=\operatorname{dim}_{\mathbf{C}} \rho_{\varpi_{6}}=27,
$$

E III $\operatorname{dim} \mathfrak{g}=78, \quad \rho_{\varpi_{1}}, \rho_{\varpi_{6}} \quad$ second kind,

$$
\operatorname{dim}_{\mathbf{C}} \rho_{\varpi_{1}}=\operatorname{dim}_{\mathbf{C}} \rho_{\varpi_{6}}=27
$$

EIV $\quad \operatorname{dim} \mathfrak{g}=78, \quad \rho_{\varpi_{1}}, \rho_{\varpi_{6}} \quad$ first kind,

$$
\operatorname{dim}_{\mathbf{C}} \rho_{\varpi_{1}}=\operatorname{dim}_{\mathbf{C}} \rho_{\varpi_{6}}=27
$$

EV $\quad \operatorname{dim} \mathfrak{g}=133, \quad \rho_{\varpi_{7}} \quad$ first kind, $\quad \operatorname{dim}_{\mathbf{C}} \rho_{\varpi_{7}}=56$,

E VI $\quad \operatorname{dim} \mathfrak{g}=133, \quad \rho_{\varpi_{7}} \quad$ second kind, $\quad \operatorname{dim}_{\mathbf{C}} \rho_{\varpi_{7}}=56$,

E VII $\operatorname{dim} \mathfrak{g}=133, \quad \rho_{\varpi_{7}} \quad$ first kind, $\quad \operatorname{dim}_{\mathbf{C}} \rho_{\varpi_{7}}=56$,

E VIII $\operatorname{dim} \mathfrak{g}=248, \quad$ none

EIX $\operatorname{dim} \mathfrak{g}=248, \quad$ none

F I $\quad \operatorname{dim} \mathfrak{g}=52, \quad \rho_{\varpi_{4}} \quad$ first kind, $\quad \operatorname{dim}_{\mathbf{C}} \rho_{\varpi_{4}}=26$,

FII $\quad \operatorname{dim} \mathfrak{g}=52, \quad \rho_{\varpi_{4}} \quad$ first kind, $\quad \operatorname{dim}_{\mathbf{C}} \rho_{\varpi_{4}}=26$,

G I $\quad \operatorname{dim} \mathfrak{g}=14, \quad \rho_{\varpi_{1}} \quad$ first kind, $\quad \operatorname{dim}_{\mathbf{C}} \rho_{\varpi_{1}}=7$.

Thus, all real non-trivial irreducible representations of $\mathfrak{g}$ of dimension less than $\operatorname{dim} \mathfrak{g}$ are determined as follows:

$$
\begin{aligned}
\mathfrak{g}= & \mathrm{EI}, \quad \rho_{\varpi_{1}}, \rho_{\varpi_{6}}, \quad \operatorname{dim}_{\mathbf{R}} \rho_{\varpi_{1}}=\operatorname{dim}_{\mathbf{R}} \rho_{\varpi_{6}}=27, \\
& \mathrm{EII}, \quad \rho_{\varpi_{1 \mathbf{R}}}, \rho_{\varpi_{6} \mathbf{R}}, \quad \operatorname{dim}_{\mathbf{R}} \rho_{\varpi_{1 \mathbf{R}}}=\operatorname{dim}_{\mathbf{R}} \rho_{\varpi_{6 \mathbf{R}}}=54,
\end{aligned}
$$


E III $, \quad \rho_{\varpi_{1 \mathbf{R}}}, \rho_{\varpi_{6} \mathbf{R}}, \quad \operatorname{dim}_{\mathbf{R}} \rho_{\varpi_{1 \mathbf{R}}}=\operatorname{dim}_{\mathbf{R}} \rho_{\varpi_{6} \mathbf{R}}=54$,

EIV $\rho_{\varpi_{1}}, \rho_{\varpi_{6}}, \quad \operatorname{dim}_{\mathbf{R}} \rho_{\varpi_{1}}=\operatorname{dim}_{\mathbf{R}} \rho_{\varpi_{6}}=27$,

$\mathrm{EV}, \quad \rho_{\varpi_{7}}, \quad \operatorname{dim}_{\mathbf{R}} \rho_{\varpi_{7}}=56$,

EVI, $\quad \rho_{\varpi_{7} \mathbf{R}}, \quad \operatorname{dim}_{\mathbf{R}} \rho_{\varpi_{7 \mathbf{R}}}=112$,

EVII, $\quad \rho_{\varpi_{7}}, \quad \operatorname{dim}_{\mathbf{R}} \rho_{\varpi_{7}}=56$,

E VIII, none

EIX, none

FI, $\quad \rho_{\varpi_{4}}, \quad \operatorname{dim}_{\mathbf{R}} \rho_{\varpi_{4}}=26$,

F II, $\quad \rho_{\varpi_{4}}, \quad \operatorname{dim}_{\mathbf{R}} \rho_{\varpi_{4}}=26$,

GI $, \quad \rho_{\varpi_{1}}, \quad \operatorname{dim}_{\mathbf{R}} \rho_{\varpi_{1}}=7$.

Comparing the dimensions of the representations of this table, it turns out that any real representation $\widetilde{V}$ of dimension $\operatorname{dim} \mathfrak{g}+1$ must contain a trivial representation, which contradicts Lemma 3.2. We have Lemma 3.5.

Lemma 3.6 Let $\mathfrak{g}$ be a real simple Lie algebra of type $A I I I_{r, s}(1 \leq r \leq$ $s, r+s=$ odd,$r+s \geq 3), \operatorname{dim} \mathfrak{g}=(r+s)^{2}-1$. Then there is no left invariant projectively flat affine connection on $G$.

Proof. All non-trivial complex irreducible representations of $\mathfrak{g}$ of dimension less than $(r+s)^{2}-1$ are as follows:

$$
\begin{array}{lll}
\rho_{\varpi_{1}}, & \rho_{\varpi_{2}}, \quad \rho_{\varpi_{r+s-2}}, \quad \rho_{\varpi_{r+s-1}}, \quad \rho_{2 \varpi_{1}}, \quad \rho_{2 \varpi_{r+s-1}} \text { and } \\
\rho_{\varpi_{3}}, \quad \rho_{\varpi_{r+s-3}} \text { only when } 6 \leq r+s \leq 8 .
\end{array}
$$

Here their dimensions are $\operatorname{dim}_{\mathbf{C}} \rho_{\varpi_{i}}=\left(\begin{array}{c}r+s \\ i\end{array}\right)$ and $\operatorname{dim}_{\mathbf{C}} \rho_{2 \varpi_{1}}=$ $\operatorname{dim}_{\mathbf{C}} \rho_{2 \varpi_{r+s-1}}=\frac{1}{2}(r+s)(r+s+1)$. Among them, $\rho_{\varpi_{k}}$ with $r+s=2 k$, $k+r=$ even, are of the first kind, and the remains are of the second kind. Therefore, all non-trivial real irreducible representations of $\mathfrak{g}$ of degree $<(r+s)^{2}-1$ are as follows:

(i) If $(r, s) \neq(2,2),(1,5),(3,3)$,

$\rho_{\varpi_{1} \mathbf{R}}, \rho_{\varpi_{r+s-1} \mathbf{R}} ; \quad \operatorname{dim}_{\mathbf{R}} \rho_{\varpi_{1} \mathbf{R}}=\operatorname{dim}_{\mathbf{R}} \rho_{\varpi_{r+s-1} \mathbf{R}}=2(r+s)$,

$\rho_{\varpi_{2} \mathbf{R}}, \rho_{\varpi_{r+s-2} \mathbf{R}} ; \quad \operatorname{dim}_{\mathbf{R}} \rho_{\varpi_{2} \mathbf{R}}=\operatorname{dim}_{\mathbf{R}} \rho_{\varpi_{r+s-2} \mathbf{R}}$

$$
=(r+s)(r+s-1) \text {. }
$$


(ii) If $(r, s)=(2,2)$,

$$
\begin{array}{ll}
\rho_{\varpi_{1}} \mathbf{R}, \rho_{\varpi_{3} \mathbf{R}} ; \quad \operatorname{dim}_{\mathbf{R}} \rho_{\varpi_{1} \mathbf{R}}=\operatorname{dim}_{\mathbf{R}} \rho_{\varpi_{3} \mathbf{R}}=2(r+s)=8, \\
\rho_{\varpi_{2}} ; & \operatorname{dim}_{\mathbf{R}} \rho_{\varpi_{2}}=6 .
\end{array}
$$

(iii) If $(r, s)=(1,5),(3,3)$,

$$
\begin{aligned}
& \rho_{\varpi_{1}} \mathbf{R}, \rho_{\varpi_{5} \mathbf{R}} ; \quad \operatorname{dim}_{\mathbf{R}} \rho_{\varpi_{1} \mathbf{R}}=\operatorname{dim}_{\mathbf{R}} \rho_{\varpi_{5} \mathbf{R}}=2(r+s)=12, \\
& \rho_{\varpi_{2} \mathbf{R}}, \rho_{\varpi_{4} \mathbf{R}} ; \quad \operatorname{dim}_{\mathbf{R}} \rho_{\varpi_{2} \mathbf{R}}=\operatorname{dim}_{\mathbf{R}} \rho_{\varpi_{4} \mathbf{R}}=(r+s)(r+s-1) \\
& =30 \text {, } \\
& \rho_{\varpi_{3}} ; \quad \quad \operatorname{dim}_{\mathbf{R}} \rho_{\varpi_{3}}=20 \text {. }
\end{aligned}
$$

Assume that $\widetilde{V}$ is the desired representation of degree $(r+s)^{2}$. Then it is a direct sum of the above representations with multiplicities. But comparing their dimensions, it turns out that the case $r+s=2 k$ (even) may only occur, and we have Lemma 3.6.

Remark 3.7 We remark here the case of real simple Lie algebra of type $A I I I_{r, s}$ with $r+s=2 k$ (even). In this case, it may only occur that

$$
\widetilde{V}=\rho_{\varpi_{i} \mathbf{R}} \oplus \cdots \oplus \rho_{\varpi_{j}} \mathbf{R} \quad(k \text {-times }),
$$

where $(i, j)=(1,1),(1,2 k-1),(2 k-1,2 k-1)$. Then,

$$
\widetilde{V}=\rho_{\varpi_{i} \mathbf{R}} \oplus \cdots \oplus \rho_{\varpi_{j} \mathbf{R}}=\mathbf{C}^{2 k} \oplus \cdots \oplus \mathbf{C}^{2 k}, \quad(k \text {-times }),
$$

where $\mathfrak{g}=\mathfrak{s u}(r, s)$ acts on $\mathbf{C}^{2 k}$ by

$$
\rho_{\varpi_{1}}(X) v=X v, \quad \text { or } \quad \rho_{\varpi_{2 k-1}}(X) v=-{ }^{t} X v
$$

for $X \in \mathfrak{s u}(r, s)$ and $v \in \mathbf{C}^{2 k}$, and $\mathbf{C}^{2 k}$ is regarded as a real vector space of dimension $4 k$. We do not know whether this $\widetilde{V}$ satisfies $(\#)$.

Lemma 3.8 Let $\mathfrak{g}$ be a real simple Lie algebra of type $B I_{r, s},(1 \leq r \leq$ $s, r+s \geq 5$, odd) except $(r, s)=(3,4)$. Then there does not exist a left invariant projectively flat affine connection on $G$.

Proof. Let $\ell=\frac{1}{2}(r+s-1)$. The fundamental complex irreducible representations $\rho_{\varpi_{i}}, i=1, \ldots, \ell-1$, are of the first kind and of complex dimension $\left(\begin{array}{c}r+s \\ i\end{array}\right)$, and $\rho_{\varpi_{\ell}}$ is the spin representation of dimension $2^{\ell}$, which is the first kind if and only if $\left[\frac{1}{2}(\ell-r+1)\right]$ is even, where $[x]$ is the integral part 
of a real number $x$. Note that $\operatorname{dim} \mathfrak{g}=\ell(2 \ell+1)$. The proof goes in a similar way as Lemma 3.6 except $(r, s)=(3,4)$ due to Theorem 3.4.

Remark 3.9 In the case $(r, s)=(3,4), \rho_{\varpi_{1}}$ and $\rho_{\varpi_{3}}$ are of the first kind and $\operatorname{dim}_{\mathbf{R}} \rho_{\varpi_{1}}=7, \operatorname{dim}_{\mathbf{R}} \rho_{\varpi_{3}}=8$. It may occur that $\widetilde{V}=\rho_{\varpi_{1}} \oplus \rho_{\varpi_{1}} \oplus \rho_{\varpi_{3}}$.

Lemma 3.10 Let $\mathfrak{g}$ be a real simple Lie algebra of type $D I_{r, s},(1 \leq r \leq$ $s, r+s \geq 8$, even). Then there does not exist a left invariant projectively flat affine connection on $G$ exept the cases $(r, s)=(1,9),(5,5),(3,11),(7,7)$.

Proof. Let $\ell=\frac{1}{2}(r+s)$. The fundamental representations $\rho_{\varpi_{i}}, i=$ $1, \ldots, \ell-2$, are of the first kind of complex dimension $\left(\begin{array}{c}2 \ell \\ i\end{array}\right)$, and $\rho_{\varpi_{\ell-1}}$ and $\rho_{\varpi_{\ell}}$ are half spin representations of complex dimension $2^{\ell-1} \cdot \rho_{\varpi_{\ell-1}}$ and $\rho_{\varpi_{\ell}}$ are both of the first kind if and only if either $r \leq s-4$ and $s-r=4 k$ with even $k$, or $r=s \geq 4$. The proof goes in a similar way as Lemma 3.6 except $\mathfrak{o}(1,9), \mathfrak{o}(5,5)(\ell=5)$, and $\mathfrak{o}(3,11), \mathfrak{o}(7,7)(\ell=7)$.

Remark 3.11 In the exceptional cases, $\rho_{\varpi_{4}}, \rho_{\varpi_{5}}$ are of the first kind for $\mathfrak{o}(1,9), \mathfrak{o}(5,5)$, and $\rho_{\varpi_{6}}, \rho_{\varpi_{7}}$ are of the first kind for $\mathfrak{o}(3,11), \mathfrak{o}(7,7)$. It may occur that $\tilde{V}=\rho_{\varpi_{1}} \oplus \rho_{\varpi_{1}} \oplus \rho_{\varpi_{1}} \oplus \rho_{\varpi_{4}}$, or $\tilde{V}=\rho_{\varpi_{1}} \oplus \rho_{\varpi_{1}} \oplus \rho_{\varpi_{1}} \oplus \rho_{\varpi_{5}}$ for $\mathfrak{o}(1,9), \mathfrak{o}(5,5)$, and $\widetilde{V}=\rho_{\varpi_{1}} \oplus \rho_{\varpi_{1}} \oplus \rho_{\varpi_{6}}$, or $\widetilde{V}=\rho_{\varpi_{1}} \oplus \rho_{\varpi_{1}} \oplus \rho_{\varpi_{7}}$ for $\mathfrak{o}(3,11), \mathfrak{o}(7,7)$.

Lemma 3.12 Let $\mathfrak{g}$ be a real simple Lie algebra of type $D I I I_{\ell}, \ell=2 r+1$ $(r \geq 2)$, or $\ell=2 r(r \geq 3)$. Then there exists no left invariant projectively flat affine connection on $G$.

Proof. In the case $\ell=2 r+1, \rho_{\varpi_{1}}, \rho_{\varpi_{2 r}}$ and $\rho_{\varpi_{2 r+1}}$ are of the second kind. In the case $\ell=2 r, \rho_{\varpi_{1}}$ and $\rho_{\varpi_{2 r-1}}$ are of the second kind, and $\rho_{\varpi_{2 r}}$ is of the first kind. Note that $\operatorname{dim} \mathfrak{g}=\ell(2 \ell-1)$. The proof goes in a similar way as Lemma 3.6.

The proof of the cases $C I_{r}$ and $C I I_{r, s}$ is given in [1, p. 157]. Thus, we have Theorem 3.1.

Acknowledgment We would like to express our sincere gratitude to Professor S. Kaneyuki and the referee who read very carefully the first draft and pointed errors, and gave useful comments. 


\section{References}

[1] Agaoka Y., Invariant flat projective structures on homogeneous spaces. Hokkaido Math. J. 11 (1982), 125-172.

[2] Bourbaki N., Groupes et algèbres de Lie, Chapitres 4, 5 et 6. Hermann, Paris, 1968.

[3] Cartan E., Les groupes projectifs continus réels qui ne laissent invariante aucune multiplicité plane. J. Math. pures et appl. 10 (1914), 149-186.

[4] Helgason S., Differential Geometry, Lie Groups and Symmetric Spaces. Academic Press, 1978.

[5] Iwahori N., On real irreducible representations of Lie algebras. Nagoya Math. J. 14 (1959), 59-83.

[6] Kaneyuki S., On the subalgebras $\mathfrak{g}_{0}$ and $\mathfrak{g}_{e v}$ of semisimple graded Lie algebras. J. Math. Soc. Japan 45 (1993), 1-19.

[7] Kaneyuki S., The Sylvester's law of inertia in simple graded Lie algebras. (to appear).

[ 8 ] Kobayashi S. and Nagano T., On projective connections. J. Math. Mech. 13 (1964), 215-235.

[9] Matsushima H. and Okamoto K., Non-existence of torsion free flat connections on a real semisimple Lie group. Hiroshima Math. J. 9 (1979), 59-60.

[10] McKay W.G. and Patera J., Tables of Dimensions, Indices, and Branching Rules for Representations of Simple Lie Algebras. Marcel Dekker, 1981.

[11] Nomizu K. and Pinkall U., On a certain class of homogeneous projectively flat manifolds. Tohoku Math. J. 39 (1987), 407-427.

[12] Nomizu K. and Sasaki T., Affine Differential Geometry. Cambridge Univ. Press, 1994.

[13] Shima H., Homogeneous spaces with invariant projectively flat affine connections. (to appear).

[14] Takeuchi M., Real Irreducible Representations of Real Lie Algebras. Osaka Univ. Dept. Math. 1996. (Japanese)

Mathematics Laboratories

Graduate School of Information Sciences

Tohoku University

Katahira, 2-1-1, Sendai 980-8577

Japan

E-mail: urakawa@math.is.tohoku.ac.jp 